\title{
Integration and commissioning of ATLAS New Small Wheel Micromegas detectors with electronics at CERN
}

\author{
Theodoros Vafeiadis ${ }^{a, *}$ on behalf of the ATLAS muon collaboration \\ ${ }^{a}$ European Organization for Nuclear Research (CERN), Geneve, Switzerland \\ E-mail: theodoros.vafeiadis@cern.ch
}

The LHC at CERN plans to have a series of upgrades to increase its instantaneous luminosity to $7.5 \times 10^{34} \mathrm{~cm}^{-2} \mathrm{~s}^{-1}$. The luminosity increase drastically impacts the ATLAS trigger and readout data rates. The inner-most station of the ATLAS muon spectrometer, the so-called Small Wheels, will be replaced with a New Small Wheel (NSW) system, consisting of Micromegas (MM) and sTGC detectors, which is expected to be installed in the ATLAS underground cavern at the end of 2021. With the final MM quadruplets (modules) already produced from different construction sites, the integration activities of the modules into the final fully-equipped MM double-wedges, that are then installed on the wheel structure, are currently in full swing in the integration facility at CERN. One crucial part of the integration workflow is the installation, testing and validation of the on-detector electronics readout chain for a very large system with more than $2.1 \mathrm{M}$ electronic channels in total. These include 4k MM Front-End Boards (MMFE8), custom printed circuit boards each one housing eight 64-channel VMM Application Specific Integrated Circuits (ASICs) that interface with the ATLAS Trigger and Data Acquisition (TDAQ) system through $1 \mathrm{k}$ data-driver Cards (ADDC \& L1DDC, respectively). The readout chain is based on optical link technology (GigaBit Transceiver links) connecting the back-end to the front-end electronics via the Front-End LInk eXchange (FELIX), a newly-developed system that will serve as the next-generation read out driver for ATLAS. Experience and performance results from the first large-scale electronics integration tests performed at CERN on final NSW MM double-wedges, including system validation tests with cosmic-rays, are presented.

40th International Conference on High Energy physics - ICHEP2020

July 28 - August 6, 2020

Prague, Czech Republic (virtual meeting)

\footnotetext{
${ }^{*}$ Speaker
} 


\section{The ATLAS New Small Wheel project}

With the increase of the rate for the High Luminosity LHC, substantial degradation of tracking performance is expected both in efficiency and resolution in the Small Wheels (SW) region of the ATLAS detector [1]. The New Small Wheels (NSW) [2] which will replace the SW will have the desired performance and will introduce trigger information from the SW region. The new system is a combination of precision tracking and trigger detectors with excellent real-time spatial and time resolution. It will provide the muon Level-1 trigger system with online track segments of good angular resolution to confirm that the muon tracks originate from the Interaction Point (IP). Subsequently, the fake triggers in the end-cap region will be significantly reduced.

Each NSW consists of 16 sectors ( 32 for both wheels) with 16 detector planes, arranged in 2 multilayers of 8 layers each. The multilayers comprise of 4 small-strip Thin Gap Chambers (sTGC) and 4 Micromegas (MM) detector planes. The sTGC detectors will provide primarily fast trigger and high precision muon tracking under the HL-LHC conditions. The Micromegas detectors will be used for precise tracking, due to their excellent spatial resolution. The detectors are arranged in a pattern which maximizes the distance between the sTGCs of the two multilayers (sTGC - MM MM - sTGC). This improves the online track segment resolution.

\section{Micromegas for the NSW}

The resistive Micromegas detector [3] comprises of two regions, the drift/conversion and the amplification region ( $5 \mathrm{~mm}$ and $120 \mu \mathrm{m}$ respectively in the case of the NSW), separated by a thin micromesh. The cathode is at the top of the drift region and the anode or readout plane is at the bottom of the amplification region. By applying suitable high voltages to the two electrodes the primary electrons, created by the incident particles, drift towards the mesh and create electron avalanches, as shown in Figure 1. The signal is induced in the anode. The anode consists of copper strips, with a layer of resistive strips on top for spark protection.
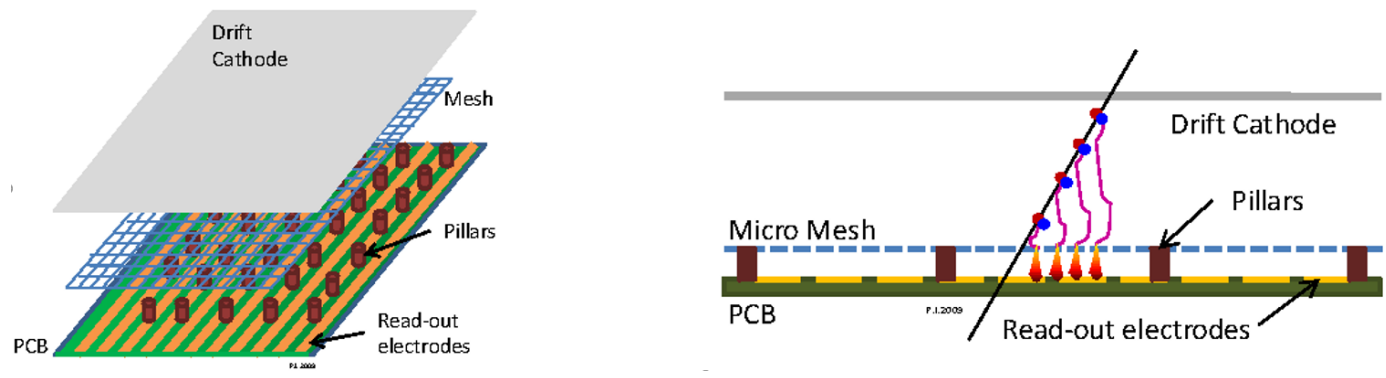

Figure 1: Schema (left) and operating principle (right) of the Micromegas detector.

In the NSW setup there are two types of sectors, the "large" and the "small", which are interchanging on the wheel to achieve the necessary overlap. Each sector is comprised by two "type 1" or "XM1" and two "type 2" or "XM2" Micromegas modules, as shown in Figure 2. Each module is called a "quadruplet" as it has four Micromegas detector planes. The quadruplets have two readout (r/o) panels, arranged in a "back-to-back" configuration. The first panel has strips parallel to the bases of the trapezoid (eta strips) for measurements on the precision coordinate (pseudorapidity $\eta$ ), 
while the two r/o planes of the second panel are tilted by $\pm 1.5^{\circ}$ (stereo strips) for measurements on the second coordinate (azimuthal). The pitch of the strips is $450(425) \mu \mathrm{m}$ for the panels in the large (small) sectors. One central double-sided panel and two external panels sustaining the stainless steel mesh (drift panels), are coupled to the two r/o panels to form the quadruplet. The assembly of the relative panels must be done with a precision better than $30 \mu \mathrm{m}$. The gas of choice for the NSW Micromegas is $93 \% \mathrm{Ar}$ and $7 \% \mathrm{CO}_{2}$.

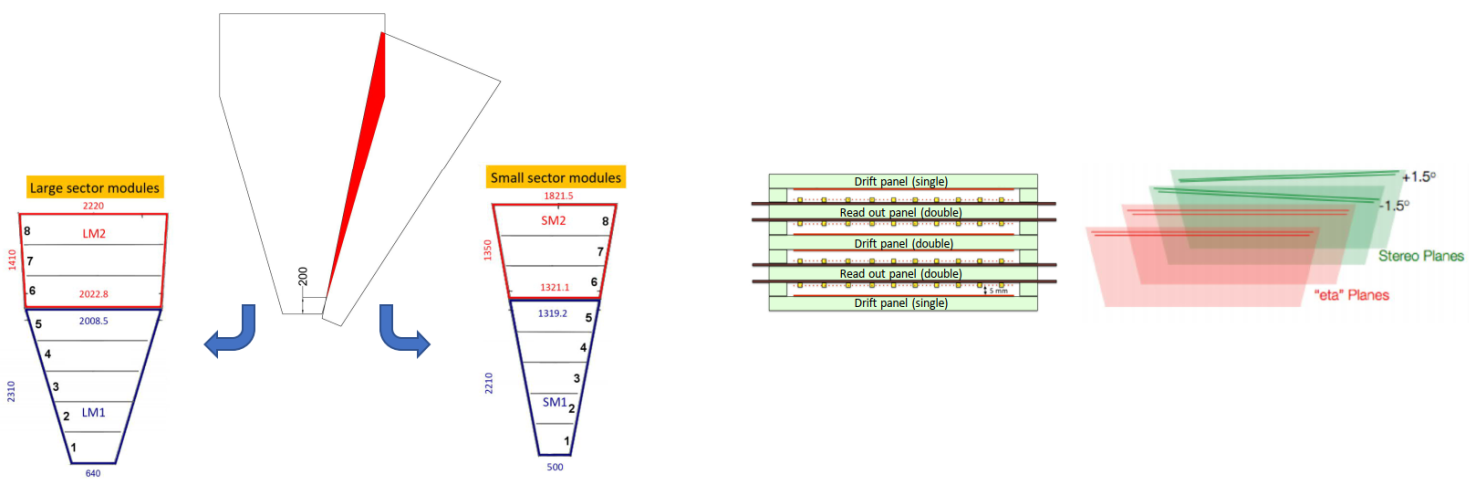

Figure 2: Left: Layout of the Micromegas sectors. Right: Sketch of the main components of a quadruplet.

\section{Micromegas Integration}

The integration of the Micromegas detectors for the NSW takes place at CERN at a large, well-equipped facility, located at building 899. It is a multistep procedure that commences with the preparation, testing and assembly of the 4 modules that comprise a Micromegas sector (2 type XM1 and 2 type XM2) with the support structure, called the Spacer Frame, as shown in Figure 3, left. The process includes the installation of the associated services for the operation of the detector, the installation and connection of the Front End Boards (FEB) and their commissioning. The final step includes validation in a cosmic stand of the completed Micromegas sector, characterisation, documentation and sign-off. Subsequently, the sector is sent off for integration with the sTGC detectors and then on the wheels.

When the individual XM1 and XM2 modules arrive they are flushed with the nominal gas mixture of $\mathrm{Ar} / \mathrm{CO}_{2}$ and they are tested for leaks. Then the first test under high voltage (HV) is performed and the HV behavior of each module is mapped. Afterwards they are transported to GIF++ [4] for irradiation tests and when they return they are prepared for the mechanical integration. In the meantime the Spacer Frame is prepared with services. When all the components are ready, the mechanical integration takes place, as shown in Figure 3, right .

The NSW has over 2 million channels read by 4096 MMFE8 [5] Front-end boards. Every 8 MMFE8 boards have 2 accompanying boards, the Level-1 Data Driver Card (L1DDC) [6] and the ART (Address in Real Time) Data Driver Card (ADDC) [7]. At each Micromegas sector 128 MMFE8, 16 ADDC and 16 L1DDC boards are installed, together with 16 Low Voltage distribution boards (LVDBs) that distribute the power to the FEBs. 

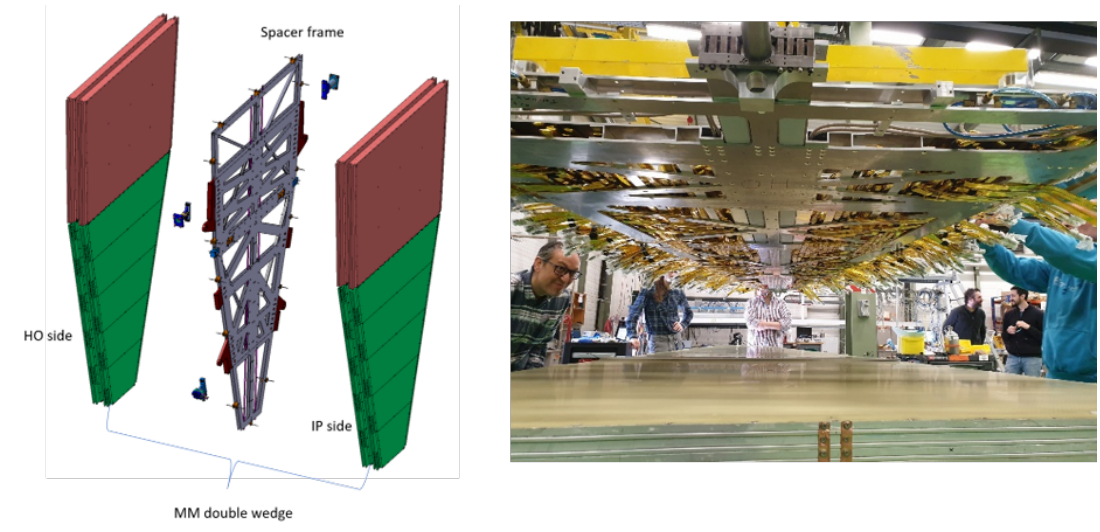

Figure 3: Left: Assembly of 4 modules (2 type XM1 and 2 type XM2) with a Spacer frame. Right: The mechanical integration of the Micromegas sectors.

The MMFE8 house 8 VMM ASICs [8] that receive the analogue signals from the detector. The zero-suppressed address, amplitude and time information is sent to both the trigger and readout path. In the trigger path the ADDC will receive the ART data (address of first arrival hit) by 8 MMFE8 boards. The first arrival hit address is selected in real time and the selected data will be sent to the trigger processor (TP). The Read-Out Controller (ROC) ASIC [9], on the MMFE8, receives the Level-1 accept (after phase 2) from the backend electronics and transmits data to the readout path. The communication with the FELIX network interface is done via the GBTx ASIC which is multiplexing a number of serial links to one single fiber, housed on the L1DDC. The L1DDC also provides the clock and bunch crossing reset to the ADDC.

The installation of the FEBs is not an easy task. The alignment of the connectors of the MMFE8 boards, with a pitch of $200 \mu \mathrm{m}$ between channels, with the pattern of the readout strips of the anode requires experience. The situation is not improved by the very limited space full of services. Three stations were installed to allow the work at 3 Micromegas sectors in a "sliding mode" and facilitate the installation and commissioning of the FEBs, as shown in Figure 4.

The mounting, alignment and connection of the FEBs takes place in Rotation Station 3. In Rotation Station 2 a full readout system has been set up. At this stage the configuration of the Slow Control adapters (SCA) [10], present to all the boards, takes place. The SCAs have three functions: to monitor operational parameters such as temperature and power consumption, to configure the ASICs of the boards and to calibrate the VMM chips of the MMFE8 boards. This step serves as a verification that the communication with the FEBs has been established. The MMFE8 boards are installed and aligned with the help of custom-built test boards. These boards have 512 LEDs, one for each strip of the detector that one MMFE8 board reads. They serve as a visual aid for the optimal position of the MMFE8, as shown in Figure 5.

After the MMFE8 boards are aligned and the communication with all FEBs is confirmed the sector is moved to the cosmic stand, shown in the left of Figure 6. The first step is to identify the noisy or dead channels of each VMM. The VMMs are set to internal pulser mode and a mapping of the noisy or dead channels is performed. MMFE8s with more than 5\% of problematic channels are replaced. The baseline and noise measurements follow, for the definition of the threshold and the adjustment of the trimmers, as shown in Figure 6, right. 


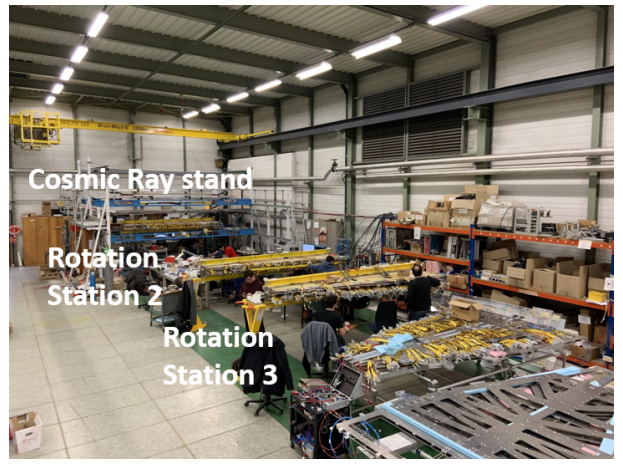

Figure 4: The three stations used for the integration and commissioning of the electronics of the Micromegas sectors.
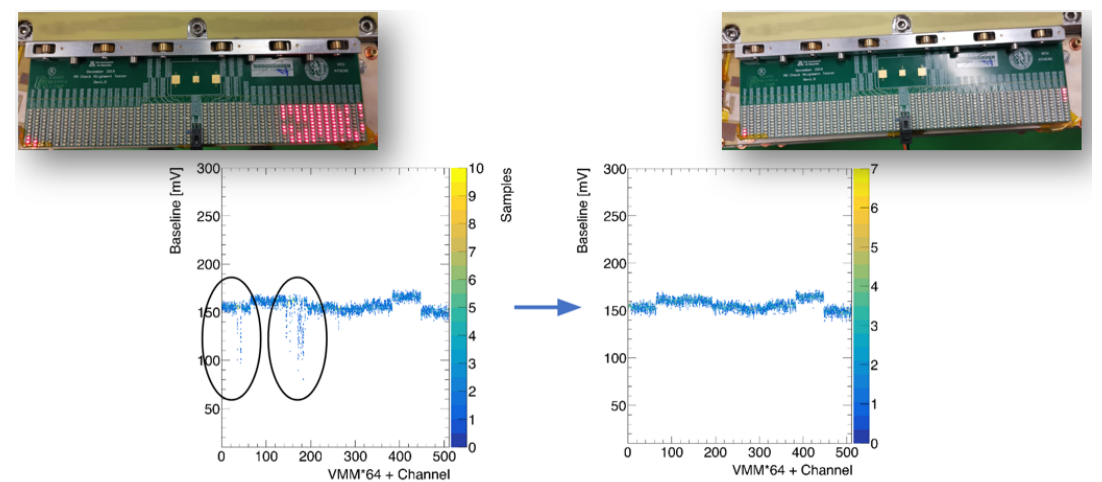

Figure 5: Baseline of the VMM chips of an MMFE8, acquired via the SCA 12-bit ADC. The misalignment of the channels appears as dips from the nominal level (circled regions, left). The correct alignment of the MMFE8 boards with the help of test boards restores the functionality of VMM channels (right).

The second step in the cosmic stand is the validation of the trigger path. All VMM channels are pulsed sequentially and the ART data are read by the TP. Ideally the map of the ART data should be uniform but some noisy or dead channels appear, as shown in the left plot of Figure 7. The percentage of dead channels for the sectors that were tested with cosmics is shown in Figure 7 , right. The final step of the electronics integration and validation is the test with cosmics. The trigger is provided by scintillators. The High Voltage sections of the detector are turned on and the final HV settings are defined. This is the final HV validation.

Each sector is required to have more than $85 \%$ of its sections working at the nominal voltage of $570 \mathrm{~V}$. The total efficiency of the sector is calculated with the cosmic run, as shown in Figure 8, and a first study on the detector characteristics is done with the data acquired.

At the end of this stage the sector is moved back to the Rotation Station 2 for the final inspection. Here the alignment corridors, the envelope, the labelling and the parenting of all the components is verified. A sign-off document is produced, which is the certificate that will accompany the sector throughout its life. The signed-off sectors are stored in a transport basket, ready to be shipped for the integration with the sTGC and finally for their installation on the NSW. 

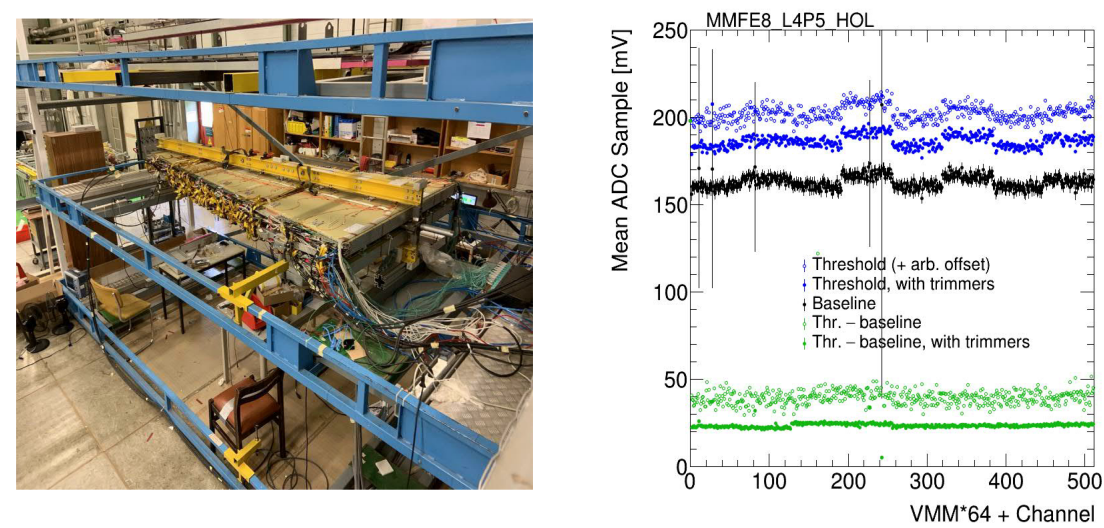

Figure 6: Left: The Cosmic Ray stand. Right: Output of a Baselines and trimmer run, example from Sector A16 : MMFE8 of Layer 4 PCB 5, HOL side.
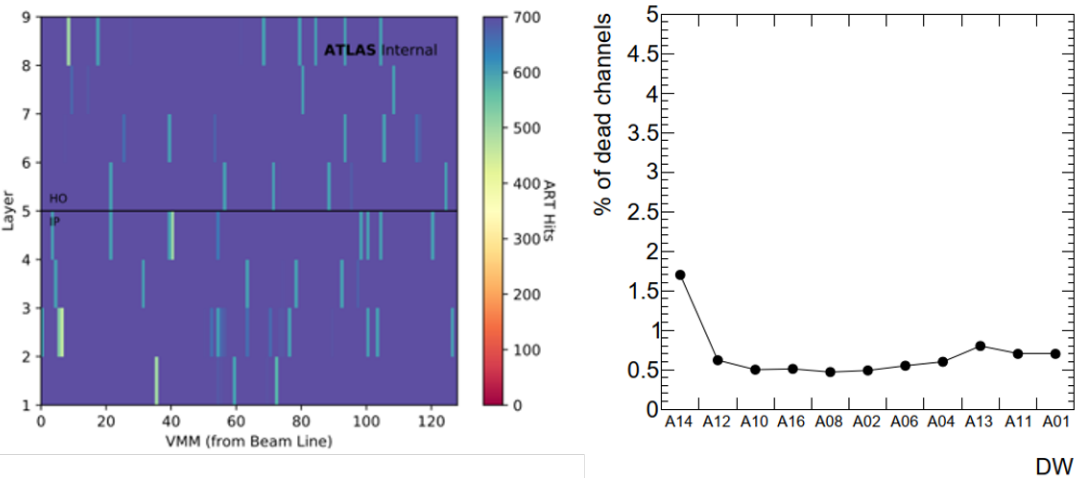

Figure 7: Left: ART map produced during the trigger path validation. Example from sector A16. Right: Percentage of dead channels per Micromegas Sector.
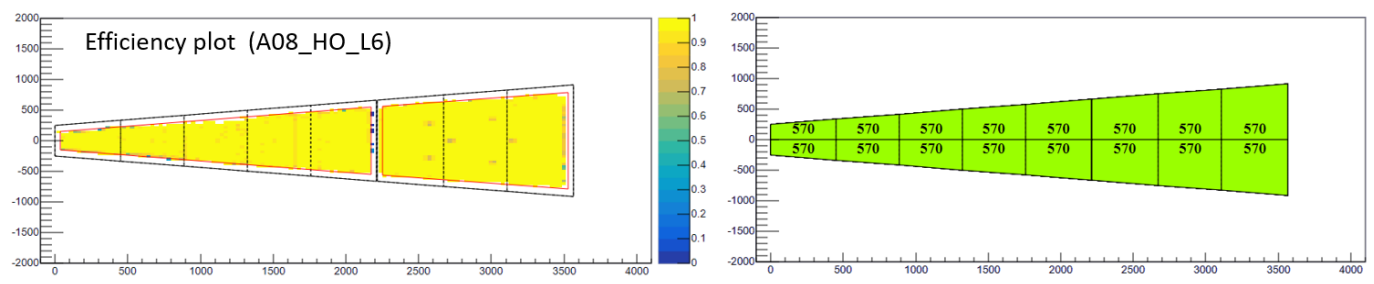

Figure 8: Left: Efficiency map, produced from a run with cosmics. Right: The HV value of the corresponding HV sections. Examples from sector A08.

\section{Conclusions}

The NSW detectors have been designed to have the desired performance to cope with high luminosity conditions after the upgrades of the LHC. The Micromegas detectors will provide the needed tracking precision in the Small Wheel region and also will significantly reduce the muon background at the first trigger level. The integration of the Micromegas modules into sectors and their commissioning and validation with cosmics is a well-defined process. A total of 8 Micromegas 
sectors for the New Small Wheel have been signed-off. A total of 7 Micromegas sectors have been integrated with the sTGC to form the NSW sectors and are installed on the wheel.

\section{References}

[1] ATLAS collaboration, The ATLAS Experiment at the CERN Large Hadron Collider, 2008 JINST 3 S08003.

[2] ATLAS collaboration, New Small Wheel Technical Design Report, CERN-LHCC-2013-006 [ATLAS-TDR-020].

[3] T. Alexopoulos et al., Performance studies of resistive-strip bulk MicrOMEGAs detectors in view of the ATLAS New Small Wheel upgrade, Nucl. Instrum. Meth. A 937 (2019) 125.

[4] M.R. Jaekel, M. Capeans, I. Efthymiopoulos, A. Fabich, R. Guida, G. Maire, M. Moll, D. Pfeiffer, F. Ravotti, H. Reithler, CERN GIF++ : A new irradiation facility to test large-area particle detectors for the high-luminosity LHC program, 2014, PoS(TIPP2014)102.

[5] P. Gkountoumis, Electronics Design and System Integration of the ATLAS New Small Wheels, ATL-MUON-PROC-2016-012.

[6] T. Alexopoulos and P. Gkountoumis, Level-1 Data Driver Card of the ATLAS New Small Wheel Upgrade Compatible with the Phase II $1 \mathrm{MHz}$ Readout Scheme, ATL-MUON-PROC2016-010.

[7] L. Yao, V. Polychronakos, H. Chen, K. Chen, H. Xu, S. Martoiu et al., The address in real time data driver card for the MicrOMEGAs detector of the ATLAS muon upgrade, 2017 JINST 12 C01047.

[8] G. Iakovidis, V. Polychronakos and G. de Geronimo, VMM - An ASIC for Micropattern Detectors, ATL-MUON-PROC-2015-015.

[9] R.M. Coliban et al., The Read Out Controller for the ATLAS New Small Wheel, ATL-MUONPROC-2015-011.

[10] A. Caratelli, S. Bonacini, K. Kloukinas, A. Marchioro, P. Moreira, R. De Oliveira et al., The GBT-SCA, a radiation tolerant ASIC for detector control and monitoring applications in HEP experiments, 2015 JINST 10 C03034. 